\title{
ON A COALESCENCE PROCESS AND ITS BRANCHING GENEALOGY
}

\author{
NICOLAS GROSJEAN, THIERRY HUILLET
}

\begin{abstract}
We define and analyze a coalescent process as a recursive boxfilling process whose genealogy is given by an ancestral time-reversed, timeinhomogeneous Bienyamé-Galton-Watson process. Special interest is on the expected size of a typical box and its probability of being empty. Special cases leading to exact asymptotic computations are investigated when the coalescing mechanisms are either linear-fractional or quadratic.
\end{abstract}

Keywords: inhomogeneous Bienyamé-Galton-Watson process; coalescence process; genealogy.

\section{INTRODUCTION}

We are interested in a model of particles iteratively coalescing at random at different sites indexing (say) energy levels and possibly leading to unoccupied sites free of particles and with no immigration of particles from outside. This can also be described as a random reallocation of balls in boxes possibly generating empty boxes, or as an iterative coarse-graining of events in a renewal process possibly generating moments free of events, or as a random walk of possibly merging particles. In such processes, there is a competition between a random force enhancing the merging of particles at some sites and a balancing random force whose effect is to generate empty sites and this trade-off is controlled by a sequence of inhomogeneous coalescing mechanisms. There is a strong analogy with the time-inhomogeneous Bienaymé-Galton-Watson (BGW) branching processes but the coalescing process is of a different nature. In fact, the genealogy of such coalescing processes after a fixed number of steps, say $N$, is a time-reversed BGW branching process conditioned on its number of offspring at the terminal value $N$. For some remarkable coalescing mechanism sequences, the expected occupancies of boxes together with the probability of a box being empty can be computed and estimated in the large $N$ limit. This concerns the linear-fractional (or $\theta$-coalescing) and the quadratic coalescing mechanisms that we shall investigate in some detail. Connections, different from ours, between coalescence Markov processes and the classical branching theory (for various branching mechanisms) appeared in [2, 3], 7] and 9], which deal with the probability that randomly sampled individuals of a time-homogeneous BGW process, alive at some generation, merge in the previous generation.

\section{A COALESCENCE PROCESS AND ITS BRANCHING GENEALOGY}

2.1. A coalescence process as a recursive box-filling process. Let $\mathbb{N}_{0}:=$ $\{0,1,2, .$.$\} and \mathbb{N}:=\{1,2, .$.$\} . We introduce a Markovian coalescence process with$ state-space $\mathbb{N}_{0}^{\mathbb{N}}$ defined as follows: consider an infinite sequence $\left\{K_{n}^{*}(i), i \geq 1\right\}$ 
giving the state of the process at step $n \in \mathbb{N}_{0} ; K_{n}^{*}(i) \in \mathbb{N}_{0}$ will be the number of balls at step $n$ in box number $i$ (or at site $i$ ), in a scenario with infinitely many boxes (sites). Suppose at step $n=0,\left\{K_{0}^{*}(i)=1, i \geq 1\right\}$ (all boxes are filled just with one ball). For each $n \geq 1$, let $\left\{M_{n}(i), i \geq 1\right\}$ be an infinite sequence of independent and identically distributed (iid) $\mathbb{N}_{0}$-valued random variables indexed by $n$, and let $f_{n}(z):=\mathbf{E}\left(z^{M_{n}}\right)$ be their common probability generating function (pgf) for which it is assumed $f_{n}(1)=1$ and $1>f_{n}(0)>0$ for each $n \geq 1$. We shall call $f_{n}(z)$ the coalescing mechanism at step $n$. Suppose $\left\{M_{n}(i), i \geq 1\right\}$ are also mutually independent across $n$.

Definition 1. The updating mechanism of $\left\{K_{n}^{*}(i), i \geq 1\right\}$ is defined as follows:

$$
K_{n+1}^{*}(i)=\sum_{m=1}^{M_{n+1}(i)} K_{n}^{*}\left(m+\sum_{j=1}^{i-1} M_{n+1}(j)\right), i \geq 1
$$

with the convention $K_{n+1}^{*}(i)=0$ if $M_{n+1}(i)=0$.

Note that a given box will never be empty for ever. We will find it also useful below to consider this dynamics stopped up to some terminal value, say $N$, of $n$. Let us comment the Markovian reallocation dynamics (1):

Suppose $\left\{K_{n}^{*}(i), i \geq 1\right\}$ are given. To compute the number of balls in box number $i$ at step $n+1$, draw a sequence $\left\{M_{n+1}(i), i \geq 1\right\}$. Fill the first box at step $n+1$ by coalescing (or merging) the balls in the $M_{n+1}(1)$ first boxes of the $n$-th step, with the convention that if $M_{n+1}(1)=0$, an empty box is created instead. This gives the size of the first box $K_{n+1}^{*}(1)$ at step $n+1$. To compute $K_{n+1}^{*}(2)$, use the remaining boxes at step $n$ with $i>M_{n+1}(1)$ and merge the $M_{n+1}(2)$ first remaining boxes. And iterate the process to form $\left\{K_{n+1}^{*}(i), i \geq 1\right\}$. From (11), it is finally clear that balls filling up some box at some step all come up from balls in some box at the preceding step: in our model of coalescence, there is no incoming balls from outside and therefore the system is closed or conservative; the adjunction of immigrants possibly filling up the empty sites at each step looks a promising issue.

Proposition 1. The $\left\{K_{n}^{*}(i), i \geq 1\right\}$ are mutually iid for each $n$. With, say, $K_{n}^{*} \stackrel{\text { law }}{=}$ $K_{n}^{*}(1)$ and $\left\{K_{n}^{*(m)}, m \geq 1\right\}$ iid copies of $K_{n}^{*}$,

$$
K_{n+1}^{*} \stackrel{\operatorname{law}}{=} \sum_{m=1}^{M_{n+1}} K_{n}^{*(m)}
$$

Proof: Due to the mutual independence $\left\{M_{n+1}(i), i \geq 1\right\}$ both in $i$ for each $n$ and then also across $n$ and because a given box at step $n$ will contribute to the construction of only one box at step $n+1$, by induction, the $\left\{K_{n}^{*}(i), i \geq 1\right\}$ are all iid for each $n$. And (2) follows from (1). Let then $\phi_{n}^{*}(z)=\mathbf{E}\left(z^{K_{n}^{*}}\right)$ be the common typical pgf of the $\left\{K_{n}^{*}(i), i \geq 1\right\}$. Then

$$
\left\{\begin{array}{l}
\phi_{n+1}^{*}(z)=f_{n+1}\left(\phi_{n}^{*}(z)\right), n \geq 0 \text { or } \\
\phi_{n}^{*}(z)=f_{n}\left(f_{n-1}\left(\ldots f_{1}(z)\right)\right), n \geq 1
\end{array},\right.
$$

gives the law of $K_{n}^{*}$ as $\mathbf{P}\left(K_{n}^{*}=j\right)=\left[z^{j}\right] \phi_{n}^{*}(z), j \geq 0$. 


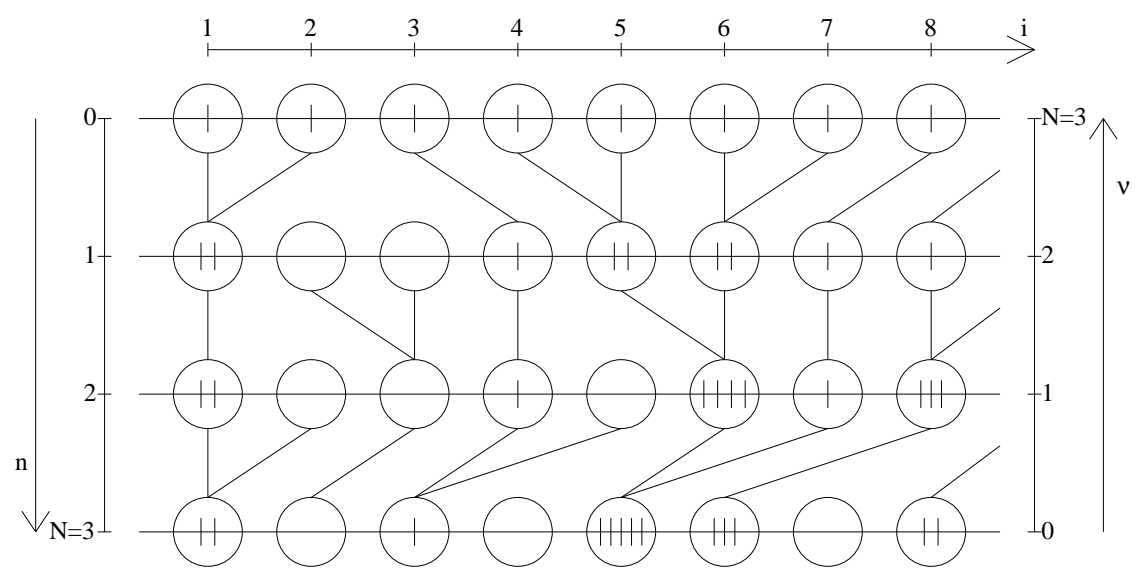

FiguRE 1. Example of a binary coalescence process with the following realizations of $M_{n}(i)$ :

$M_{1}(1)=2, M_{1}(2)=0, M_{1}(3)=0, M_{1}(4)=1, M_{1}(5)=2$,

$M_{1}(6)=2, M_{1}(7)=1, M_{1}(8)=1, \ldots$;

$M_{2}(1)=1, M_{2}(2)=0, M_{2}(3)=2, M_{2}(4)=1, M_{2}(5)=0$,

$M_{2}(6)=2, M_{2}(7)=1, M_{2}(8)=2, \ldots$

$M_{3}(1)=2, M_{3}(2)=1, M_{3}(3)=2, M_{3}(4)=0, M_{3}(5)=2$,

$M_{3}(6)=1, M_{3}(7)=0, M_{3}(8)=1, \ldots$.

Of particular interest is the probability $\mathbf{P}\left(K_{n}^{*}=0\right)=\phi_{n}^{*}(0)$ to have a typical empty box at step $n$, together with the mean size $\mathbf{E}\left(K_{n}^{*}\right)$ of the typical box at step $n$. With $n_{1}>n_{2}$, we can also define

$$
\Phi_{n_{1}, n_{2}}^{*}(z):=f_{n_{1}}\left(f_{n_{1}-1}\left(\ldots f_{n_{2}+1}(z)\right)\right) \text {. }
$$

Then, with $n_{1}>n_{2}>n_{3}, \Phi_{n_{1}, n_{3}}^{*}(z)=\Phi_{n_{1}, n_{2}}^{*}(z) \circ \Phi_{n_{2}, n_{3}}^{*}(z):=\Phi_{n_{1}, n_{2}}^{*}\left(\Phi_{n_{2}, n_{3}}^{*}(z)\right)$, a concatenation property of the time-inhomogeneous coalescent process $\left\{K_{n}^{*}\right\}$. It holds $\Phi_{n, 0}^{*}(z)=\phi_{n}^{*}(z)$.

Although the $\left\{K_{n}^{*}(i), i \geq 1\right\}$ are all iid for each $n$, for any $(i, j), K_{n}^{*}(i)$ and $K_{n+1}^{*}(j)$ are not necessarily independent: for instance if $K_{n}^{*}(1)$ is large, $K_{n+1}^{*}(1)$ is more likely to be large.

We also observe that for any finite $n, \phi_{n}^{*}(0)$ cannot be equal to 1 . Indeed, if this were the case, there would exist $n_{*} \leq n$ such that $\phi_{n_{*}}^{*}(0)=1$ and $\phi_{n_{*}-1}^{*}(0)<1$ (recalling $\left.\phi_{0}^{*}(0)=0\right)$. Now $\phi_{n_{*}}^{*}(0)=f_{n_{*}}\left(\phi_{n_{*}-1}^{*}(0)\right)$ and $f_{n_{*}}$ is invertible because $f_{n_{*}}$ is not constant equal to 1 ; therefore $\phi_{n_{*}-1}^{*}(0)=1$ which contradicts the existence of $n_{*}$ : for any $n$, all $\left\{K_{n}^{*}(i), i \geq 1\right\}$ cannot be simultaneously equal to 0 . Moreover, the sequence $\phi_{n}^{*}(0)$ is neither necessarily increasing nor decreasing with $n$ (see the examples below).

By independence of the $K_{n}^{*}(i) \mathrm{s}$, in view of $0<\phi_{n}^{*}(0)<1$, for each fixed $n \in$ $\{1, \ldots, N\}$, we also have

$$
\mathbf{P}\left(\min _{i \geq 1} K_{n}^{*}(i)>0\right)=\left(1-\phi_{n}^{*}(0)\right)^{\infty}=0
$$

(at least one box is empty) and by strong law of large numbers, $\mathbf{P}\left(K_{n}^{*}=j\right)=$ $\lim _{I \rightarrow \infty} \frac{1}{I} \sum_{i=1}^{I} \mathbf{1}_{\left\{K_{n}^{*}(i)=j\right\}}$, for any $j \geq 0$, together with $\mathbf{E}\left(K_{n}^{*}\right)=\lim _{I \rightarrow \infty} \frac{1}{I} \sum_{i=1}^{I} K_{n}^{*}(i)$, 
if this quantity exists (is finite). In particular, $\mathbf{P}\left(K_{n}^{*}=0\right)=\phi_{n}^{*}(0)$. Note finally,

$$
\lim _{I \rightarrow \infty} \frac{1}{I} \sum_{i=1}^{I} M_{n+1}(i)=\mathbf{E}\left(M_{n+1}\right)=f_{n+1}^{\prime}(1),
$$

and $\mathbf{E}\left(K_{N}^{*}\right)=\phi_{N}^{* \prime}(1)=\prod_{n=1}^{N} f_{n}^{\prime}(1)=\prod_{n=1}^{N} \mathbf{E}\left(M_{n}\right)$, if these quantities exist (are finite).

Definition 2. We shall say that we have a subcritical, critical or supercritical coalescence process if the (geometric mean) limit $\mu=\lim _{N \rightarrow \infty} \mathbf{E}\left(K_{N}^{*}\right)^{1 / N}$ exists and $\mu<1,=1,>1$, respectively. If for each $n, \mathbf{E}\left(M_{n}\right)=\infty$ so that $\mathbf{E}\left(K_{N}^{*}\right)=\infty$, we shall put $\mu:=\infty$ and the process will be said strongly supercritical.

In the (sub-)critical cases, we expect $\mathbf{P}\left(K_{N}^{*}=0\right) \underset{N \rightarrow \infty}{\rightarrow} 1$, and of interest is the rate at which $\mathbf{P}\left(K_{N}^{*}>0\right)$ tends to 0 . In the supercritical case, we expect $\mathbf{P}\left(K_{N}^{*}=0\right) \underset{N \rightarrow \infty}{\rightarrow} \rho \in[0,1)$ and of interest is when $\rho>0$ and the rates at which $\mathbf{P}\left(K_{N}^{*}>0\right)$ tends to $1-\rho \geq 0$. In this setup, we will give below one example for which $\rho \in(0,1)$ (the supercritical $\theta$-coalescing model below) and another one for which $\rho=0$ (the supercritical quadratic branching model).

In Figure 1 we give a partial view of a sample realization of the binary coalescence process. Picking the size $j=5$ node at terminal step $N=3$ and moving backwards the steps, we see a tree that goes up to step 0 with 5 leaves of size 1 . Picking the first size $j=0$ node at step $N=3$ and moving backwards, we see a tree made of empty nodes only that goes extinct at step 1 . The ancestral tree of the first size $j=1$ node at step $N=3$ has an empty node at step 2 . It goes up to the step 0 with 1 leaf of size 1 .

Remark 1. (i) If the law of $M_{n+1}(i)$ is the same for each $n \geq 0$ and $i \geq 1$ (with the obvious simplifications of the construction), we shall speak of a homogeneous coalescence process with homogeneous coalescing mechanism $f$, independent of $n$.

(ii) A slight extension of (11): let $(t(i), i \geq 1)$ be a positive sequence, possibly random and iid, representing say time lags (energy spacings or gaps). Let $s(i)=\sum_{j=1}^{i} t(j), i \geq 1$ be the moments of events (the energy levels). Defining $\left\{K_{n}^{*}(s(i)), i \geq 1\right\}$ to be the number of events (or particles) at times (or energy levels) $s(i),\left\{K_{0}^{*}(s(i))=1, i \geq 1\right\}$ is a renewal sequence of events and

$$
K_{n+1}^{*}(s(i))=\sum_{m=1}^{M_{n+1}(i)} K_{n}^{*}\left(s\left(m+\sum_{j=1}^{i-1} M_{n+1}(j)\right)\right), i \geq 1
$$

gives the way the renewal sequence is updated by iterated coalescence of events in the previous step, as a coarse-graining process possibly generating empty sites. As in (11), this process is driven by the coalescing mechanism sequences.

2.2. Genealogy as a time-reversed inhomogeneous BGW branching process. Consider a discrete-time and time-inhomogeneous BGW branching process [1], [5], 6], 10, whose reproduction laws are given by the probability systems $\mathbf{P}\left(M_{n}=m\right)=\pi_{n}(m), m, n \geq 1$ for the number $M_{n}$ of offspring per capita produced at generation $n$ to form generation $n+1$. We assume $\pi_{n}(0)>0$ so that the process can go extinct. We let $f_{n}(z)=\mathbf{E}\left(z^{M_{n}}\right)=\sum_{m \geq 0} \pi_{n}(m) z^{m}$ be the 
probability generating function of $M_{n}$ (the branching mechanism) and we assume $f_{n}(1)=1$ for each $n \geq 1$. With $\mathrm{K}_{n}$ the number of individuals alive at generation $n$ given $\mathrm{K}_{0}=1$, we have $\mathrm{K}_{n+1}=\sum_{k=1}^{\mathrm{K}_{n}} M_{n+1}^{(k)}$ where $M_{n+1}^{(k)}$ are iid copies of $M_{n+1}$ giving the offspring number of each individual of the population alive at generation $n$. Thus,

$$
\phi_{n+1}(z)=\phi_{n}\left(f_{n+1}(z)\right), \phi_{0}(z)=z
$$

leading classically to

$$
\mathbf{E}\left(z^{\mathrm{K}_{n}}\right):=\phi_{n}(z)=f_{1}\left(f_{2}\left(\ldots f_{n}(z)\right)\right), n \geq 1 .
$$

$\phi_{n}(z)$ is the $n$-th composition of the different $f_{m}(z)$ in the reverse order to the one giving $\phi_{n}^{*}$ in (3). Recall that with $n_{1}<n_{2}<n_{3}$, if

$$
\Phi_{n_{1}, n_{2}}(z):=f_{n_{1}+1}\left(f_{n_{1}+2}\left(\ldots f_{n_{2}}(z)\right)\right)
$$

then $\Phi_{n_{1}, n_{3}}(z)=\Phi_{n_{1}, n_{2}}(z) \circ \Phi_{n_{2}, n_{3}}(z):=\Phi_{n_{1}, n_{2}}\left(\Phi_{n_{2}, n_{3}}(z)\right)$, the standard concatenation property of the time-inhomogeneous BGW process $\left\{\mathrm{K}_{n}\right\}$.

When running the above branching process $\left\{\mathrm{K}_{n}\right\}$ backward in time starting from a terminal generation say $n=N$ to $n=0$ for any given $N>0$, we get a new (truncated) branching process say $\left\{K_{\nu}, 0 \leq \nu \leq N\right\}$ defined as follows:

Definition 3. The process $\left\{K_{\nu}, 0 \leq \nu \leq N\right\}$ is defined by its pgf at generation number $\nu: \mathbf{E}\left(z^{K_{0}}\right)=z$ and

$$
\mathbf{E}\left(z^{K_{\nu}}\right):=\Phi_{N, N-\nu}^{*}(z)=f_{N}\left(f_{N-1}\left(\ldots f_{N-\nu+1}(z)\right)\right), 1 \leq \nu \leq N
$$

so with reversed pgf sequence $g_{1}=f_{N}, \ldots, g_{\nu}=f_{N-\nu+1}, \ldots, g_{N}=f_{1}$.

This suggests that $\left\{K_{n}^{*}, 0 \leq n \leq N\right\}$ and $\left\{K_{\nu}, 0 \leq \nu \leq N\right\}$ could be time-reversed of one another (in a different sense to the time-reversal defined in [4), with the latter being the ancestral process of the former. Note in this respect that $\nu$ in Figure 1 runs from bottom to top of the graph, starting from $\nu=0$.

The relation between the cluster coalescence process $\left\{K_{n}^{*}, 0 \leq n \leq N\right\}$ considered up to a given terminal generation $N$ and the BGW process $\left\{K_{\nu}, 0 \leq \nu \leq N\right\}$ in (5), as time-reversed processes, is indeed as follows:

Theorem 2. At given terminal step $N>1$ of the process $\left\{K_{n}^{*}(i), i \geq 1\right\}$, pick any box number $i$ and suppose it has size $j$, an event occurring with probability $\mathbf{P}\left(K_{N}^{*}=j\right)$. Then the ancestral tree of this size $j$ box, running backward in $n$ (so forward in $\nu$ ), will be the one of a $B G W$ process as in (5), started from a single individual (the selected box) but conditioned on having $j$ descendants at generation $\nu=N$.

Proof: (i) The creation of an empty box at step $N$, running forward in $n$, is understood as the extinction before time $N$ of its ancestral tree as a time-reversed BGW process. This empty box has indeed no non-empty offspring (parents) at generation $N-1$ although it can have one or more empty boxes offspring. If the randomly chosen box is empty, its ancestral tree is therefore made of empty nodes only and its height will be strictly less than $N$. It is the one of a time-reversed BGW process started from a single individual and conditioned on having no descendants (extinction) at generation $\nu=N$, meaning $K_{N}=0$.

(ii) The coalescence of $m \geq 1$ boxes into one single box forward in $n$ at step $N$ is understood as this box giving birth to $m$ offspring at step $N-1$, backward in $n$ 
(forward in $\nu$ ), one of which at least being non empty. If the randomly chosen box is non-empty (of size $j>0$ ) indeed, the content of this box (its size) is read from its number of ancestors at generation $N$ : its BGW ancestral tree has exactly $j$ leaves at generation $\nu=N$ and it will have height $N$. We conclude for instance that the law of the number $K_{\nu}, 1 \leq \nu \leq N$, of ancestors at generation $\nu$ of a selected size $j$ box at step $N$ produced by the coalescence process (1), is given from (5) by

$$
\mathbf{P}\left(K_{\nu}=k \mid K_{N}=j\right)=\frac{\left[z_{1}^{k} z_{2}^{j}\right] \Phi_{N, N-\nu}^{*}\left(z_{1} \Phi_{N-\nu, 0}^{*}\left(z_{2}\right)\right)}{\left[z_{2}^{j}\right] \Phi_{N, 0}^{*}\left(z_{2}\right)}
$$

recalling $\mathbf{P}\left(K_{\nu}=k, K_{N}=j\right)=\left[z_{1}^{k} z_{2}^{j}\right] \Phi_{\nu, 0}^{*}\left(z_{1} \Phi_{N, \nu}^{*}\left(z_{2}\right)\right)$. As required, we have

$$
\begin{aligned}
\sum_{j \geq 0} \mathbf{P}\left(K_{N}^{*}\right. & =j) \mathbf{E}\left(z_{1}^{K_{\nu}} \mid K_{N}=j\right)=\sum_{j \geq 0}\left[z_{2}^{j}\right] \phi_{N}^{*}\left(z_{2}\right) \frac{\left[z_{2}^{j}\right] \Phi_{N, N-\nu}^{*}\left(z_{1} \Phi_{N-\nu, 0}^{*}\left(z_{2}\right)\right)}{\left[z_{2}^{j}\right] \Phi_{N, 0}^{*}\left(z_{2}\right)} \\
& =\sum_{j \geq 0}\left[z_{2}^{j}\right] \Phi_{N, N-\nu}^{*}\left(z_{1} \Phi_{N-\nu, 0}^{*}\left(z_{2}\right)\right)=\Phi_{N, N-\nu}^{*}\left(z_{1} \Phi_{N-\nu, 0}^{*}(1)\right) \\
& =\Phi_{N, N-\nu}^{*}\left(z_{1}\right)=f_{N}\left(f_{N-1}\left(\ldots f_{N-\nu+1}\left(z_{1}\right)\right)\right)=\mathbf{E}\left(z_{1}^{K_{\nu}}\right) .
\end{aligned}
$$

Remark 2. Here is another connection of $\left\{K_{n}^{*}, 0 \leq n \leq N\right\}$ with the BGW process $\left\{K_{\nu}, 0 \leq \nu \leq N\right\}$ : in the civil system of kinship degree (see for example [1]), the degree of kinship between two individuals of the same family is the number of edges between these individuals (an individual is related to itself by a degree 0). The law of the number of individuals of the generation $N$ that are related to some individual at the same generation by a degree of kinship at most $2 n$ is the same as the one of a coalescence process $K_{n}^{*}$.

\section{EXAMPLES}

There are classes of discrete branching and/or coalescence processes for which the $\operatorname{pgf} \phi_{N}(z)$ of $\mathrm{K}_{N}$ and/or the $\operatorname{pgf} \phi_{N}^{*}(z)$ of $K_{N}^{*}$ is exactly computable, thereby making the above computations concrete and somehow explicit, in the large $N$ limit. One is related to the linear-fractional branching mechanism [8] (so called here $\theta$ branching and that we shall rename $\theta$-coalescing in view of our time-reversal), the other one being the quadratic coalescing mechanism which we shall define.

3.1. The $\theta$-coalescing mechanism. With $|\theta| \leq 1, a_{n}, b_{n}>0$, consider the $\theta$ coalescing mechanism model, defined by

$$
\left\{\begin{array}{c}
f_{n}(z)=z_{c}-\left(a_{n}\left(z_{c}-z\right)^{-\theta}+b_{n}\right)^{-1 / \theta} \text { or } \\
\left(z_{c}-f_{n}(z)\right)^{-\theta}=a_{n}\left(z_{c}-z\right)^{-\theta}+b_{n},
\end{array}\right.
$$

and for those values of $a_{n}, b_{n}>0$ and $z_{c} \geq 1$, for which $f_{n}$ is a pgf with $f_{n}(1)=1$. This is an inhomogeneous version of a pgf family defined in [8].

Proposition 3. This family is stable under composition. Indeed,

$$
\phi_{n}^{*}(z)=f_{n}\left(\ldots f_{1}(z)\right)=z_{c}-\left(A_{n}^{*}\left(z_{c}-z\right)^{-\theta}+B_{n}^{*}\right)^{-1 / \theta}
$$


is a pgf with $A_{n}^{*}=\prod_{m=1}^{n} a_{m}$ and $B_{n}^{*}=B_{n}^{*}=b_{n}+\ldots+b_{k} \prod_{l=k+1}^{n} a_{l}+\ldots+b_{1} \prod_{l=2}^{n} a_{l}$. We also have

$$
\mathbf{P}\left(K_{N}^{*}=0\right)=\phi_{N}^{*}(0)=z_{c}-\left(A_{N}^{*} z_{c}^{-\theta}+B_{N}^{*}\right)^{-1 / \theta}
$$

and if the means exist, $f_{n}^{\prime}(1)=a_{n}\left(a_{n}+b_{n}\left(z_{c}-1\right)^{\theta}\right)^{-(1+\theta) / \theta}=a_{n}$ because $f_{n}(1)=$ 1 entails $b_{n}=\left(z_{c}-1\right)^{-\theta}\left(1-a_{n}\right)$. Thus,

$$
\mathbf{E}\left(K_{N}^{*}\right)=\phi_{N}^{* \prime}(1)=\prod_{n=1}^{N} f_{N}^{\prime}(1)=A_{N}^{*} \text { with } \mathbf{E}\left(K_{N}^{*}\right)^{1 / n}=A_{N}^{* 1 / N} .
$$

For the sake of simplicity, we shall limit ourselves in the following examples to the cases $z_{c}=1$ and $\theta \in\{-1,0,1\}$.

3.1.1. $\theta=-1$ (affine case). Here $f_{n}(z)=1-\left(a_{n}(1-z)+b_{n}\right)$ and $f_{n}(1)=1$ imposes $b_{n}=0$ and $a_{n} \in(0,1)$. We have

$$
\phi_{n}^{*}(z)=1-A_{n}^{*}+A_{n}^{*} z .
$$

- Take for example $a_{n}=\mu\left(1-1 /(n+1)^{\alpha}\right)$ with $\alpha>0$.

If $\alpha=1: \mathbf{E}\left(K_{N}^{*}\right)=\prod_{n=1}^{N} f_{n}^{\prime}(1)=\prod_{n=1}^{N} a_{n}=a^{N} /(n+1)$ so that $\mathbf{E}\left(K_{N}^{*}\right)^{1 / N} \rightarrow$ $\mu=a$. If $\mu=1(\mu<1)$ we get a critical (subcritical) coalescence process. Here, $\mathbf{P}\left(K_{N}^{*}=0\right)=\phi_{N}^{*}(0)=1-\prod_{n=1}^{N} a_{n}=1-\mu^{N} /(N+1) \rightarrow 1$. Asymptotically, the typical box gets empty with probability 1 , at rate $\mu^{N} /(N+1)$ and $\mathbf{P}\left(K_{N}^{*}=0\right)$ is increasing with $N$ for the affine model.

If $\alpha>1: \mathbf{E}\left(K_{N}^{*}\right)^{1 / N} \rightarrow \mu=a$. And $\mathbf{P}\left(K_{N}^{*}=0\right)=\phi_{N}^{*}(0)=1-\prod_{n=1}^{N} a_{n}=$ $1-\mu^{N} \frac{\zeta(\alpha)-1}{N} \rightarrow 1$, where $\zeta(\alpha)$ is the zeta function.

If $\alpha<1: \mathbf{P}\left(K_{N}^{*}=0\right)=\phi_{N}^{*}(0)=1-\prod_{n=1}^{N} a_{n}=1-\frac{1}{(1-\alpha)} N^{-\alpha} \mu^{N} \rightarrow 1$.

- More generally, take $a_{n}=a\left(1-\varepsilon_{n}\right)$ where $\varepsilon_{n} \in[0,1)$ and $\varepsilon_{n} \underset{n \rightarrow \infty}{\rightarrow} 0$. Then, it holds that the Cesàro mean $\left(\sum_{m=1}^{n} \varepsilon_{m}\right) / n \underset{n \rightarrow \infty}{\rightarrow} 0$. Thus $\mathbf{E}\left(K_{N}^{*}\right)^{1 / N} \rightarrow \mu=a$ and $\mathbf{P}\left(K_{N}^{*}=0\right) \rightarrow 1$, the rates of which depending on the way $\varepsilon_{n} \underset{n \rightarrow \infty}{\rightarrow} 0$.

3.1.2. $\theta=1$ (standard homographic case). Here $f_{n}(z)$ takes the form

$$
f_{n}(z)=\left(1-\frac{1}{a_{n}+b_{n}}\right)+\frac{1}{a_{n}+b_{n}} \frac{z}{1+b_{n} / a_{n}(1-z)},
$$

where $a_{n}, b_{n}>0$ and $a_{n}+b_{n}>1$. We have

$$
\phi_{n}^{*}(z)=1-\frac{1}{A_{n}^{*}+B_{n}^{*}}+\frac{1}{A_{n}^{*}+B_{n}^{*}} \frac{z}{1+B_{n}^{*} / A_{n}^{*}(1-z)},
$$

where $A_{n}^{*}, B_{n}^{*}>0$ and $A_{n}^{*}+B_{n}^{*}>1$. We have $f_{n}^{\prime}(1)=1 / a_{n}$ and taking $a_{n}=$ $a\left(1-\varepsilon_{n}\right)$ where $\varepsilon_{n} \underset{n \rightarrow \infty}{\rightarrow} 0, \mathbf{E}\left(K_{N}^{*}\right)=\prod_{n=1}^{N} f_{n}^{\prime}(1)=\prod_{n=1}^{N} a_{n}^{-1}$ so that $\mathbf{E}\left(K_{N}^{*}\right)^{1 / N} \rightarrow$ $\mu=1 / a$. Depending on $\mu<1, \mu=1$ or $\mu>1$, we get a subcritical, critical or supercritical coalescence process. Moreover,

$$
\mathbf{P}\left(K_{N}^{*}=0\right)=\phi_{N}^{*}(0)=1-\frac{1}{A_{N}^{*}+B_{N}^{*}}
$$

now depends on the choice of the sequence $b_{n}>1-a_{n}$. 
Taking $b_{n}=b\left(1-a_{n}\right)$ with $b>1$ if $a_{n}<1$ or $b<1$ if $a_{n}>1$, so that $a_{n}+b_{n}>1$ always, we get telescoping sums leading to $B_{N}^{*}=b\left(1-A_{n}^{*}\right)$. Thus,

$$
\mathbf{P}\left(K_{N}^{*}=0\right)=\frac{(b-1)\left(1-A_{N}^{*}\right)}{A_{N}^{*}+b\left(1-A_{N}^{*}\right)}<1, b \lessgtr 1, \text { if } A_{N}^{*} \gtrless 1 .
$$

If $a>1$ (a subcritical case because $\mu<1$ ), take for example $a_{n}=a\left(1+\varepsilon_{n}\right)$ where $\varepsilon_{n}>0$ and $\varepsilon_{n} \underset{n \rightarrow \infty}{\rightarrow} 0$ fast enough. We get $\mathbf{P}\left(K_{N}^{*}=0\right) \rightarrow 1$ and with $A_{N}^{*-1 / N} \rightarrow 1 / a: \mathbf{P}\left(K_{N}^{*}>0\right) \sim \frac{1}{1-b} A_{N}^{*-1} \rightarrow 0$.

If $a<1$ (a supercritical case because $\mu>1$ ), take for example $a_{n}=a\left(1-\varepsilon_{n}\right)$ where $\varepsilon_{n} \in(0,1)$ and $\varepsilon_{n} \underset{n \rightarrow \infty}{\rightarrow} 0$ fast enough. We get $\mathbf{P}\left(K_{N}^{*}=0\right) \rightarrow \rho=$ $(b-1) / b>0$ and with $A_{N}^{* 1 / N} \rightarrow a: \rho^{-1} \mathbf{P}\left(K_{N}^{*}=0\right) \sim 1-\frac{1}{b} A_{N}^{*} \rightarrow 1$.

3.1.3. $\theta=0$ (infinite mean case). Let $a_{n} \in(0,1)$. The case $\theta=0$ is defined by continuity by $f_{n}(z)=1-\left(1-\rho_{n}\right)^{1-a_{n}}(1-z)^{a_{n}}$, provided $b_{n}$ is given by $\rho_{n}=$ $1-\left(\left(1-a_{n}\right) / b_{n}\right)^{1 / \theta} \in(0,1)$. Here $f_{n}^{\prime}(1)=\infty$. Let $\lambda_{n}:=\left(1-\rho_{n}\right)^{1-a_{n}}$ and consider then $f_{n}(z)=1-\lambda_{n}(1-z)^{a_{n}}$. It holds that

$$
\phi_{N}^{*}(z)=1-\Lambda_{N}^{*}(1-z)^{A_{N}^{*}},
$$

where $\Lambda_{N}^{*}=\prod_{n=1}^{N} \lambda_{n}^{\prod_{m=n+1}^{N} a_{m}}$. Thus, $\mathbf{E}\left(K_{N}^{*}\right)=\infty$ (strong supercriticality of such a coalescence process) and

$$
\mathbf{P}\left(K_{N}^{*}=0\right)=\phi_{N}^{*}(0)=1-\Lambda_{N}^{*} .
$$

We can produce an example for which $\mathbf{P}\left(K_{N}^{*}=0\right) \underset{N \rightarrow \infty}{\rightarrow} \rho>0$ and control its speed of convergence. Take $a_{n}=a\left(1-\varepsilon_{n}\right)$ with $\varepsilon_{n} \rightarrow 0$ fast enough $(a \in(0,1))$ and suppose $\lambda_{n}=\lambda^{1-a_{n}}$ where $\lambda \in(0,1)$. Then

$$
1-\phi_{N}^{*}(z)=\lambda\left(\frac{1-z}{\lambda}\right)^{A_{N}^{*}}
$$

showing that (again because $A_{N}^{* 1 / N} \rightarrow a$ )

$$
\mathbf{P}\left(K_{N}^{*}=0\right)=\phi_{N}^{*}(0) \sim 1-\lambda^{1-A_{N}^{*}} \rightarrow 1-\lambda=: \rho>0 .
$$

If for instance $\varepsilon_{n} \rightarrow 0$ like $n^{-\alpha}$ with $\alpha>1, \mathbf{P}\left(K_{N}^{*}>0\right) \rightarrow \lambda=1-\rho>0$ at double exponential speed: $\lambda^{-1} \mathbf{P}\left(K_{N}^{*}>0\right) \sim \lambda^{-C a^{n}}$, for some constant $C>0$.

3.2. The quadratic coalescing mechanism. Consider the quadratic map: $f(z)=$ $a(b+z)^{2}-b$. We have $f(z)=h^{-1}(g(h(z)))$ where $h(z)=z+b, g(z)=a z^{2}$. For such maps $f$,

$$
f^{\circ 0}(z):=z \text { and } f^{\circ n}(z)=a^{2^{n}-1}(b+z)^{2^{n}}-b, n \geq 1
$$

and the $n$-th iterates $f^{\circ n}(\cdot)=f(f(\ldots f(\cdot)))$ ( $n$ times) of $f$ can explicitly be found as a degree $2^{n}$-polynomial. Note that $\left(f^{\circ n}\right)^{-1}(z)=f^{\circ-n}(z)$.

Can $f(z)$ be a non trivial (ie different from the purely quadratic map $a z^{2}$ ) pgf so that $f^{\circ n}(z)$ would be a pgf for all $n$ ? For this to hold, we should have $a, b>0$ and $a b \geq 1$ but also $f(0) \in(0,1)$ and $f(1)=1$. The latter conditions require that $a=1 /(b+1)$ which cannot be fulfilled together with $a b \geq 1$. So if $a, b>0$ and $a b \geq 1, f(z)$ is a quadratic map with positive $\left[z^{k}\right]$-coefficients, $k=0,1,2$, but these summing to a constant larger than one. So these coefficients cannot be 
probabilities and neither such a $f$ nor its $n$-th iterate can be a genuine pgf. Observe that the $\left[z^{k}\right]$-coefficients of $f^{\circ n}(z)$ are also non-negative in this case for all $n$.

We nevertheless have the following result:

Proposition 4. $\phi_{n}^{*}(z)=f^{\circ n}(z) / f^{\circ n}(1)$, as given from (14), is the explicit pgf of a time-inhomogeneous coalescing Markov process whose generating coalescing mechanism is

$$
f_{n+1}(z)=\frac{\left(a b+\left((a(b+1))^{2^{n}}-a b\right) z\right)^{2}-a b}{(a(b+1))^{2^{n+1}}-a b} .
$$

Proof: suppose that $f$ is chosen with $a, b>0$ and $a b \geq 1$ so that it is absolutely monotone on $(0,1)$. Consider the $\operatorname{pgf} \phi_{n}^{*}(z)=f^{\circ n}(z) / f^{\circ n}(1)$, normalizing $f^{\circ n}(z)$ so as $\phi_{n}^{*}(1)=1$, leading to

$$
\phi_{n}^{*}(z)=\frac{a^{2^{n}-1}(b+z)^{2^{n}}-b}{a^{2^{n}-1}(b+1)^{2^{n}}-b}=\frac{[a(b+z)]^{2^{n}}-a b}{[a(b+1)]^{2^{n}}-a b} .
$$

We have $\phi_{0}^{*}(z)=z$ and

$$
\phi_{n+1}^{*}(z)=f^{\circ n+1}(z) / f^{\circ n+1}(1)=\frac{f\left(f^{\circ n}(1) \phi^{\circ n}(z)\right)}{f\left(f^{\circ n}(1)\right)}=: f_{n+1}\left(\phi_{n}^{*}(z)\right),
$$

where $f_{n+1}(z):=\frac{f\left(f^{\circ n}(1) z\right)}{f\left(f^{\circ n}(1)\right)}$ is an inhomogeneous pgf, with $f^{\circ n}(1)=a^{2^{n}-1}(b+1)^{2^{n}}-$ $b, f\left(f^{\circ n}(1)\right)=f^{\circ n+1}(1)$, explicitly known. The inhomogeneous binary coalescing mechanism of such a Markov process reads

$$
\begin{aligned}
f_{n+1}(z) & =\frac{f\left(\left(a^{2^{n}-1}(b+1)^{2^{n}}-b\right) z\right)}{a^{2^{n+1}-1}(b+1)^{2^{n+1}}-b}=\frac{a\left(b+\left(a^{2^{n}-1}(b+1)^{2^{n}}-b\right) z\right)^{2}-b}{a^{2^{n+1}-1}(b+1)^{2^{n+1}}-b} \\
& =\frac{a^{2}\left(b+\left(a^{2^{n}-1}(b+1)^{2^{n}}-b\right) z\right)^{2}-a b}{a^{2^{n+1}}(b+1)^{2^{n+1}}-a b}=\frac{\left(a b+\left((a(b+1))^{2^{n}}-a b\right) z\right)^{2}-a b}{(a(b+1))^{2^{n+1}}-a b} .
\end{aligned}
$$

Note $f_{n+1}(z) \underset{n \rightarrow \infty}{\rightarrow} z^{2}$ because $a(b+1)>1$ : the asymptotic inhomogeneous driving pgf is purely quadratic (a purely binary coalescence process) with $f_{n+1}(0)=$ $a b(a b-1) /\left((a(b+1))^{2^{n+1}}-a b\right) \rightarrow 0$, very fast.

For such a binary coalescing process, we have:

(i) $\phi_{N}^{* \prime}(1)=\mathbf{E}\left(K_{N}^{*}\right)=\frac{a^{2^{N}-1} 2^{N}(b+1)^{2^{N}}-1}{a^{2^{N}-1}(b+1)^{2^{N}}-b} \sim \frac{2^{N}}{b+1} \rightarrow \infty$. Thus $\mathbf{E}\left(K_{N}^{*}\right)^{1 / N} \rightarrow \mu=$ $2>1$. The binary coalescent process is supercritical.

(ii) Concerning the probability of an empty box, $\mathbf{P}\left(K_{N}^{*}=0\right)=\phi_{N}^{*}(0)=$ $\left((a b)^{2^{N}}-a b\right) /\left((a(b+1))^{2^{N}}-a b\right) \underset{N \rightarrow \infty}{\sim}(b /(b+1))^{2^{N}} \rightarrow \rho=0$. The sequence $\phi_{N}^{*}(0)=\mathbf{P}\left(K_{N}^{*}=0\right)$ is decreasing very fast with $N$ (at double exponential speed) and so $K_{n}^{*}=0 \nRightarrow K_{n+1}^{*}=0$ : the coalescent process can hit 0 where it can be regenerated. In addition, for $j \in\left\{0, \ldots, 2^{n}\right\}$,

$$
\mathbf{P}\left(K_{n}^{*}=j\right)=\left[z^{j}\right] \phi_{n}^{*}(z)=\frac{a^{2^{n}-1}}{a^{2^{n}-1}(b+1)^{2^{n}}-b}\left(\begin{array}{c}
2^{n} \\
j
\end{array}\right) b^{2^{n}-j}
$$


gives the full distribution of $K_{n}^{*}$.

Acknowledgments: TH acknowledges support from the "Chaire Modélisation mathématique et biodiversité". NG and TH also acknowledge support from the labex MME-DII Center of Excellence (Modèles mathématiques et économiques de la dynamique, de l'incertitude et des interactions, ANR-11-LABX-0023-01 project). The authors are indebted to J. Avan for careful reading of the manuscript.

\section{REFERENCES}

[1] Athreya, K. B. and Ney, P. Branching Processes. Springer, New York, 1972.

[2] Athreya, K. B. Coalescence in critical and subcritical Galton-Watson branching processes. J. of Appl. Prob. 49, 627-638, (2012).

[3] Athreya, K. B. Coalescence in the recent past in rapidly growing populations. Stochastic Processes and their Applications, vol. 122, issue 11, 3757-3766, (2012).

[4] Esty, W. The reverse Galton-Watson process, Journal of Applied Probability, 12, 574-680, (1975).

[5] Harris, T. E. The theory of branching processes. Die Grundlehren der Mathematischen Wissenschaften, Bd. 119 Springer-Verlag, Berlin; Prentice-Hall, Inc., Englewood Cliffs, N.J. 1963.

[6] Jagers, P. Galton-Watson processes in varying environments. J. Appl. Probability, 11, 174$178,(1974)$.

[7] Lambert, A. Coalescence times for the branching process. Adv. in Appl. Probab., Volume 35, Number 4, 1071-1089, (2003).

[8] Sagitov S. and Lindo A. A special family of Galton-Watson processes with explosions. In Branching Processes and Their Applications. Lect. Notes Stat. Proc. (I.M. del Puerto et al eds.) Springer, Berlin, 2016 (to appear). arxiv.org/pdf/1502.07538, (2015).

[9] Sheth, R. K. Galton-Watson branching processes and the growth of gravitational clustering. Mon. Not. R. Astron. Soc. 281, 1277-1289, (1996).

[10] Tuljapurkar, S. Population Dynamics in Variable Environments. Berlin and New York: Springer-Verlag, 1990

[11] https://www.umanitoba.ca/faculties/arts/anthropology/tutor/descent/cognatic/civil.html

Laboratoire de Physique Théorique et Modélisation, CNRS-UMR 8089 et Université de Cergy-Pontoise,, 2 Avenue Adolphe Chauvin, 95302, Cergy-Pontoise, France, EMAIL: Nicolas.GrosjeAn@U-CERGy.Fr, ThIERRY.HuILLET@U-CERGy.FR 